\title{
Implications of Model Compliance Program for Interventional Pain Physicians
}

\author{
William A. Sarraille, JD
}

The model compliance program for small physician practices, recently released by the Office of the Inspector General (OIG) shows a remarkable degree of flexibility reflective of the OIG's acknowledgment that small physician practices have such limited resources that they might not be in a position to implement a full compliance program.

The OIG suggests a step by step approach to the implementation of a compliance program which includes auditing and monitoring, establishing practice standards and procedures, designating na compliance officer or contact, conducting training and education, responding to offenses and corrective actions, opening uses of communication, and enforcing discipline through well published guidelines. The OIG's final plan also lists a wide variety of risk areas that a small practice should address in its program, many of the identified risk areas relate to situations in which physicians interact with other types of providers-including durable medical equipment suppliers, home health agencies, clinical laboratories, hospitals, and others. Although physicians have tended to equate the term "compliance" with "billing and coding" compliance, the OIG's list of risk areas reveals a much broader range of compliance issues and concerns, with particular emphasis on financial relationships between physicians and other providers.

In conclusion, Physician practices are clearly at greater risk today if they fail to do so in light of the publication of the OIG's final plan.

Keywords: Office of Inspector General (OIG), compliance program, billing and coding, fraud and abuse
The Office of the Inspector General for the Department of Health and Human Services (OIG) recently released a final model compliance program for small physician practices. The model plan shows a remarkable degree of flexibility reflective of the OIG's acknowledgment that small physician practices have such limited resources that they might not be in a position to implement a full compliance program. The OIG's final model plan incorporates many items suggested by the American Society of Interventional Pain Physicians (ASIPP) in written comments submitted by ASIPP to the agency.

\section{AN “OPERATIONAL" APPROACH}

In the past, OIG has stressed what it expects "out" of a voluntarily implemented compliance program, rather than how an organization can get "there" as a matter of process. Traditionally, the OIG has defined an "effective com-

From Arent Fox Klintner Plotkin and Kahn, PLLC, Washington, DC. William A. Sarraille is a partner in the Health Law group of Arent Fox and general counsel to American Society of Interventional Pain Physicians. William A. Sarraille, JD, 1050 Connecticut Avenue NW, Washington, DC 20036-5339. Email: sarailw@arentfox.com pliance program" by stating simply that it must include:

- a compliance officer and/or committee to direct the program, with the appropriate authority and direct access to the decision-makers in the organization,

- written standards of conduct and a written compliance plan,

- the development and use of corrective action procedures,

- compliance training,

- periodic monitoring and auditing,

- disciplinary protocols, and

- the screening of employees and contractors to ensure that they are not inappropriately delegated responsibility for sensitive functions.

This list of the necessary "outputs," though, does not tell providers anything about "how" to go about putting a compliance program in place.

${ }^{1}$ If your practice is like most small (or frankly large) practices, your "standards and procedures" review won't take very long-because you don't have a comprehensive policies and procedures manual. 
Realizing that the "how" question is particularly important from the small physician practice perspective (because physician practices are more likely to feel overwhelmed in the absence of very practical advice from an operational point of view), the OIG took the unprecedented step in the final physician plan by emphasizing the outline of a compliance process, instead of a list of required end products. The OIG deserves a significant degree of praise for taking this approach, which ASIPP encouraged the OIG to follow. OIG also should be commended for recognizing that the process used by small practices may very well not lead to all of the elements of a traditional "effective compliance program" being implemented-simply because of resource, personnel, and time limitations.

The OIG suggests the following approach to the implementation of a compliance program in a small physician practice. Although the OIG's step by step approach will be the right process for some practices to adopt, it will not, as the OIG itself appears to concede, be the correct sequence for others. Blind adherence to the OIG's model would be ill-advised.

\section{STEP ONE: AUDITING AND MONITORING}

The first step that the OIG mentions is to audit and monitor the current situation. OIG believes that this can be accomplished by doing two things: (1) a review of the practice's existing "standards and procedures" and (2) a "claims review."

In undertaking a claims review, the OIG stresses four areas of testing:

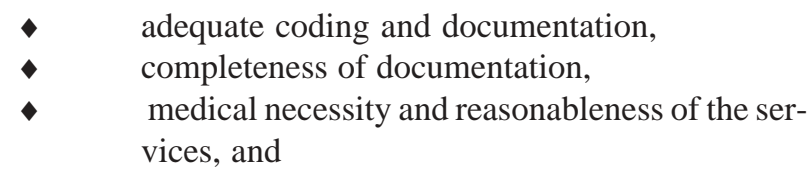

${ }^{2}$ This is one of the places in the guidance where OIG appears to have been a bit too flexible in the standards it set. In an interventional pain management practice, at least, five services per year is clearly inadequate to determine whether or not a provider is coding correctly the range of services that are provided in this kind of practice. Given that interventional pain physicians provide or order E\&M services, consultations, and a wide variety of ancillary diagnostic and therapeutic services, in addition to the interventional procedures themselves, a minimum of twenty services per year seems more practical. a check to determine the presence of "incentives" for the over-utilization of services.

The OIG says that the review may be either prospective (claims not yet submitted) or retrospective (claims already submitted) in nature. The OIG states that the results of the review should be used as a benchmark, to assess compliance the success of compliance efforts on an on-going basis.

The OIG also writes that auditing and monitoring must have continuing application. At a minimum, annual audits must be undertaken. Reviews of no less than five services per provider should be completed. ${ }^{2}$ The OIG cautions that negative audit results must lead to corrective action. According to the OIG, the appropriate response to an identified problem will depend on the circumstances. "In some cases," the OIG states, "it can be as straight-forward as generating a repayment with appropriate explanation." "In others," the OIG suggests, "the practice may want to consult with a billing/coding expert to determine the next best action."

\section{STEP TWO: PRACTICE STANDARDS AND PROCEDURES}

OIG says that the next step that a small physician practice should take is to develop written practice standards and procedures. The standards and procedures should address any areas of vulnerability identified in the baseline audit. Calling the standards and procedures "a central component of a compliance program," the OIG writes that small practices may look to programs developed by medical societies and associations, such as ASIPP, or by contractual partners, such as a physician practice management company, a management services organization, or the local hospital where services are provided. However, a practice should not just adopt a model program wholesale and simply install the "off the shelf" product - a point made in ASIPP's own model plan. The small practice's plan must be tailored to its needs and circumstances.

The OIG suggests that small practices consider developing a compliance binder that would include:

written standards addressing the risk areas identified by OIG for small practices (more about those later), attachments consisting of OIG fraud alerts and advisory opinions, as well as the applicable Medicare bulletins, and 
$\bullet$

clinical forms designed to secure appropriate and complete documentation.

The binder must be reasonably accessible to members of the practice and its staff. OIG encourages practices to train their new employees on the contents of the compliance binder "as soon as possible" as part of their orientation.

\section{STEP THREE: COMPLIANCE OFFICER OR CONTACT}

The OIG says that the next step that a small practice should consider is who will direct the compliance program on an on-going basis. Although "ideally" a single person will be selected as the compliance officer and will be responsible for the compliance program as a whole, the OIG acknowledges in its model plan that this might not be possible in a small practice with limited resources and personnel. Accordingly, OIG states that it is "acceptable" to "spread" the compliance functions over a number of "compliance contacts," so long as the division of responsibility is made clear in the practice's written plan.

The OIG's final plan also indicates that practices can "share" compliance officers retained on an independent contractor or other basis. The final plan's discussion of this option, however, is somewhat muted, as compared to the discussion of this same concept in the OIG's earlier proposed model. Critics, including ASIPP, had responded to this proposal raising a host of concerns - ranging from issues of privacy and confidentiality, the whistle-blower implications, and the potential anti-trust concerns. Although the final plan does not address most of these concerns, OIG does state that a shared compliance officer can create concerns if the compliance officer is so disengaged from a practice that he or she has no real connection to it. In other words, though it is often important to ensure that a compliance officer can act with independence, a compliance officer can be too detached to be effective.

Addressing other "sharing" issues, the OIG appears to have no particular difficulty with a physician practice using a hospital plan as a guide in developing its own program, or in making use of compliance training offered by a hospital. The OIG does express the concern, however, about a hospital's support of other physician compliance activities, including audits, unless the institution will be compensated at fair market value for those services. The OIG's concern is based on the Federal Anti-
Kickback Statute. The OIG has apparently determined that there is a risk that hospitals will use free compliance services to reward or induce referrals in violation of the statute. Presumably, the OIG would have the same reaction to free compliance services offered by ambulatory surgery centers, as it would to those offered by hospitals.

Critics, including ASIPP, have questioned the wisdom of the OIG's position in this area. Institutions, like hospitals and ambulatory surgery centers, often have difficulty improving their compliance, because they are largely dependent on independent physicians for a number of critical decisions that affect compliance. Institutional providers can demonstrate the need for changes in behavior that will improve the compliance of both the practice and institution by providing free or discounted billing and coding audits for practices. Some critics do not see any particular anti-kickback risks where these services are provided to all physicians on staff who request them, not just "big referrers."

\section{STEP FOUR: TRAINING AND EDUCATION}

According to the OIG, the next step that a small practice should consider taking is the implementation of a program of training and education. "Ideally" the training will consist of both what the OIG refers to as "general" and "specific" training. General training consists of training on the practice's compliance standards and procedures, including the "nuts and bolts" of how the practice's compliance program operates. Specific training consists of training on particular compliance risk areas, like the waiver of co-payments and deductibles.

The OIG suggests that small practices create their training and education programs by answering three questions. First, the practice needs to determine who needs training-both general and specific. The implicit assumption in that statement, of course, is that not all practice personnel necessarily need compliance training. This would be the case, for instance, with very low-level employees who have no role in billing, coding, documentation or other substantive issues.

The second question is how should the training be delivered. The OIG states that training can be presented in live seminars or through newsletters, self-study, or inservices. Although this sounds as though the OIG is open to training programs that do not include any "live" sessions, the OIG cautions that training and education programs must be "effective." 
The final question is when and how often should training be conducted. The OIG states that "at a minimum" training should occur on an annual basis.

In terms of topics for training, the OIG makes a number of recommendations. Training should include education on the practice's compliance program generally (and how it operates), including the means to report issues and concerns and the discipline that will be imposed where violations are found to exist. Two major goals of compliance training should be (1) to show employees how to conduct their responsibilities in compliance with federal law and (2) how compliance is a condition of employment. The OIG also recommends training on relevant statutes and regulations. The OIG provides a number of relevant legal authorities in an appedix to its final plan.

The OIG suggests that specific and "extensive" training on billing and coding issues be provided to all those involved "directly" in billing. Training topics should include:

- claims development and submission processes,

- the legal issues posed in signing materials for a physician without the physician's authorization,

- proper documentation of services,

- appropriate billing and coding standards and procedures, and

- the legal sanctions for deliberately or recklessly filing false claims.

Effective education includes having up-to-date reference materials, such as CPT and ICD-9 code books and carrier bulletins. Training on billing and coding issues may be obtained, the OIG notes, from community colleges, carriers, and professional associations.

\section{STEP FIVE: CORRECTIVE ACTIONS}

In the words of the OIG, "upon receipt of reports or reasonable indications or suspected noncompliance, it is important that the compliance contact or officer look into the allegations to determine whether a significant violation of applicable law or the requirements of the compliance program has indeed occurred, and to take decisive steps to correct the problem." The OIG stresses that, depending on the circumstances, refunds or disclosures may be required. OIG states its expectation that criminal violations will be reported to the government. Known overpayments, even in the absence of criminal wrong-doing, should result in repayments to the applicable payor. When problems are identified, it is important that the practice take steps quickly to ensure that the problem is not magnified or compounded.

In order to facilitate appropriate corrective action, the OIG suggests that practices create a variety of "monitors and warning indicators." These might include systems to check for:

significant changes in the types of claims rejec-
tions,
correspondence from carriers or intermediaries
challenging the medical necessity of services or
the validity of claims,
illegal or unusual patterns in code utilization,
and
high volumes of unusual payment adjustments.

\section{STEP SIX: COMMUNICATION}

In other plans addressed to other types of providers prior to the issuance of the final small physician plan, the OIG had stressed the need for compliance programs to include a means of anonymous reporting of compliance issues, preferably through a telephone hotline. In announcing this component of the small physician plan, however, the OIG accepted that anonymity may simply not be possible in a small physician practice. Although OIG states its belief that e-mail or telephone anonymous reporting can be achieved by larger practices, OIG writes that an "open door" policy should be sufficient for small practices.

An open door policy consists of:

- mandatory reporting of conduct a reasonable person would believe to be erroneous or fraudulent,

- creation of a "user-friendly" process for reporting issues,

- the creation of a standard that makes the failure to report compliance issues grounds for instituting discipline, and

the creation of a simple and readily accessible mechanism for processing compliance reports.

The practice must also implement and enforce a policy that the practice will not tolerate any retribution made for reporting a compliance concern in good faith.

An open door policy should also involve a clear system for effective communication between a small practice and 
a third party billing agent, to the extent the practice contracts with one. Such a system should include communication regarding:

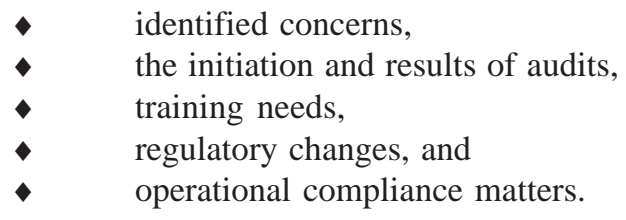

\section{STEP SEVEN: DISCIPLINARY PROTOCOLS}

Despite criticism from many organizations, including ASIPP, that an emphasis on disciplinary protocols is inconsistent with the type of voluntary and collaborative process that compliance is supposed to entail, the OIG insists in its final plan that "consistent and appropriate sanctions" are a "necessary" component of an effective compliance program. They are necessary, OIG says, to "add credibility," if for no other reason. Inclusion of disciplinary guidelines in the practice's written standards and procedures is sufficient to meet the OIG's expectation that the standards related to discipline be "well-publicized."

\section{RISK AREAS}

The OIG's final plan lists a wide variety of risk areas that a small practice should address in its program. The tremendous number of risk areas, collected in an appendix to the final plan, are a source of concern for a number of commentators, including ASIPP. who are afraid that small physician practices will be overwhelmed by the laundry list of risk areas that they may elect not to create a compliance program. If that were to occur, the OIG will have hurt its own effort to create an environment of self-policing in physician services.

Interestingly, many of the identified risk areas relate to situations in which physicians interact with other types of providers - including durable medical equipment suppliers, home health agencies, clinical laboratories, hospitals, and others - who have been under the fraud and abuse microscope for a long time. Many of the listed items correspond to areas that the OIG has addressed previously in special fraud alerts and in advisory opinions. Although physicians have tended to equate the term "compliance" with "billing and coding" compliance, the OIG's list of risk areas reveals a much broader range of compliance issues and concerns, with particular emphasis on financial relationships between physicians and other providers.
Organized under four principal risk area headings, the OIG enumerated the following risk areas:

\section{Coding and Billing Issues}

- $\quad$ Billing for items or services not provided or not provided as claimed;

- $\quad$ Submitting claims for equipment, supplies, or services that are not reasonable and necessary;

- Double billing ;

- Billing for non-covered services as if they were covered services;

- $\quad$ Knowing misuse of provider numbers that result in improper payments;

- Unbundling;

- $\quad$ Failure to use coding modifiers appropriately;

- $\quad$ Clustering;

- Inappropriate use of advanced beneficiary notices (also known as waiver of liability forms);

- $\quad$ Problems related to the reassignment of the right to bill or collect; and

- Upcoding.

\section{Ensuring Reasonable and Necessary Services}

- $\quad$ Following local medical review policy restrictions;

- $\quad$ Appreciating and abiding by Medicare restrictions on the coverage of screening services;

- Inappropriate use and completion of certificates of medical necessity; and

- Not submitting non-covered services except to secure a denial for purposes of secondary coverage requirements.

\section{Accurate and Complete Documentation of Various Elements}

- $\quad$ The site of service;

- $\quad$ Appropriateness of the services;

- $\quad$ Accuracy of the billing;

- Identities of the care-givers; and

- Design of appropriate systems to ensure a legible and complete record showing:

- $\quad$ The reason for the encounter;

- "Any" relevant history;

- $\quad$ Physical examination findings; 
- $\quad$ Prior [presumably relevant] diagnostic test results;

- The assessment, clinical impression, or diagnosis;

- The plan of care;

- $\quad$ Date and legible identity of the observer;

- The reasons for the ordering of diagnostic testing if those reasons are not "easily inferred" from the record;

- $\quad$ CPT and ICD-9 codes supported by the record;

- $\quad$ Correct use of coding modifiers;

- Accurate linkage of CPT codes to ICD9 codes;

- Provision of accurate information to the Medicare program regarding other insurance coverages, where known;

- $\quad$ Appropriate documentation of participation in care and supervision of care under the teaching physician rules; and The identification of appropriate health risk factors, including the patient's progress, his or her responses to treatment, and any revisions in diagnoses.

- Improper Inducements, Kickbacks, and Self-Referrals

- $\quad$ Financial relationships with hospitals, hospices, nursing facilities, home health agencies, and pharmaceutical companies, and others who are in a referral relationship (including non-federal program relationships because they can have an indirect effect on federal program referrals);

- $\quad$ Arrangements that do not reflect fair market value;

- Joint ventures with individuals or entities providing goods or services to the practice or its patients;

- $\quad$ Consulting contracts or medical directorships;

Office and equipment leases with entities in referral relationships;

Compliance with the OIG's restrictions

on physician-hospital cost savings "gainsharing";

Percentage compensation arrangements in third party billing contracts;
- $\quad$ Appropriate professional courtesy prac- tices; and
- Solicitation of any gift or gratuity of more than nominal value.

\section{DISCUSSION}

As indicated earlier, commentators have roundly praised the OIG for showing a real measure of flexibility in its development of the final model plan. The final plan clearly states that, if a practice does not have sufficient resources, it can implement a compliance mechanism that represents something less than a full compliance program as set out in the definition of an "effective compliance program" that is contained in the United States Sentencing Commission Guidelines.

Despite this, however, some have questioned whether the OIG has really committed to recognizing the compliance efforts of small physician practices that do not implement a full compliance program. The OIG's discussion of this issue leaves it with room to argue that particular practices who fail to fully implement a compliance program did not abide by the agency's guidance.

For instance, at one point in the final model plan document, the OIG states that "full implementation of a compliance program may not be feasible for all physician practices." [Emphasis added]. The OIG also writes, at one point, that "some physicians may not fully implement all of the components of a [compliance] program." [Emphasis added]. The OIG also states that the "extent of implementation will depend on the size and resources of the practice." [Emphasis added].

The negative pregnant of these and similar statements may be that the OIG will dispute the sufficiency of compliance mechanisms, in False Claims Act or other enforcement actions, if the agency concludes that the practice could (and should) reasonably have done more. For large practices, the OIG states its expectation that those entities will combine the physician practice model compliance program and the OIG's third party billing company model compliance program to develop a "complete" compliance program.

The situation is made all the more unclear and frustrating by the OIG's studied refusal to define a "small physician practice." Despite repeated requests from medical societies, such as ASIPP, the OIG has not offered any real guidance on how to distinguish between small and large 
physician practices, other than to simply state that "small" practices are those that have the "financial and staffing resources that would prevent the implementation of a full scale, institutionally-structured compliance program." In a most unhelpful response to the repeated requests for guidance on this central question, the OIG has defended its failure to provide any instruction with the statement that the difference between a small and a large physician practice "cannot be determined by stating a particular number."

On a positive note, the OIG's final model program responds to the criticism made by many medical societies, including ASIPP, that the original draft program incorrectly implied that compliance programs were required of physician groups. The OIG flatly states that "these Guidelines are not mandatory."

With that said, ASIPP and some other medical societies have expressed their concern with the failure of the draft plan to stress the responsibilities of others to work with physician practices in resolving the ambiguities currently endemic in the Medicare, Medicaid and other government-funded health care programs. Specifically, ASIPP called upon the OIG to stress its own responsibility and that of the Health Care Financing Administration, the Medicare carriers, the Medicare intermediaries and others to work to address these issues. ASIPP was particularly focused on this issue given the variable and inconsistent instructions issued by carriers and intermediaries on a host of interventional pain management procedure coding issues. Unfortunately, without even commenting on this request made by ASIPP and other medical societies, the final model plan fails to discuss in any significant way the responsibilities of governmental agencies in the compliance process, other than their enforcement powers. The final plan also includes some reassuring words for physicians regarding the intent of the OIG in the exercise of its enforcement powers. The OIG states, for instance, that the False Claims Act is designed only to target intentional, knowing, or at least reckless conduct, not mere negligent errors or "honest mistakes." For many, though, those reassuring words ring hollow, as the dividing line between a "reckless" act and a merely "negligent" one is often in the eye of the beholder. The misuse of a single CPT code repeated over a period of time will be viewed by the federal government as a reckless pattern of misconduct, even though, from the provider's perspective, it represents a single, honest mistake in the evaluation of a single code.

The model plan also attempts to respond to the criticism of some that compliance program activities can have the unintended effect of distracting physicians from patient care activities. The OIG specifically acknowledges in the final plan that "patient care is and should be the first priority" for physicians. According to the OIG, however, "patient focus can be enhanced" through compliance activities.

\section{CONCLUSION}

Only time will tell whether the OIG's efforts at announcing what it viewed as flexible guidance will encourage more practices to implement at least some compliance mechanisms. Physician practices are clearly at greater risk today if they fail to do so in light of the publication of the OIG's final plan. 